$J H A$, xxxvi (2005)

\title{
THE EPOCH OF THE CONSTELLATIONS ON THE FARNESE ATLAS AND THEIR ORIGIN IN HIPPARCHUS'S LOST CATALOGUE
}

\author{
BRADLEY E. SCHAEFER, \\ Louisiana State University, Baton Rouge
}

\section{BACKGROUND}

The Farnese Atlas is a Roman statue depicting the Titan Atlas holding up a celestial globe that displays an accurate representation of the ancient Greek constellations (see Figures 1 and 2). This is the oldest surviving depiction of this set of the original Western constellations, and as such can be a valuable resource for studying their early development. The globe places the celestial figures against a grid of circles (including the celestial equator, the tropics, the colures, the ecliptic, the Arctic Circle, and the Antarctic Circle) that allows for the accurate positioning of the constellations. The positions shift with time due to precession, so the observed positions on the Farnese Atlas correspond to some particular date. Also, the declination of the Arctic and Antarctic Circles will correspond to a particular latitude for the observer whose observations were adopted by the sculptor. Thus, a detailed analysis of the globe will reveal the latitude and epoch for the observations incorporated in the Atlas; and indeed these will specify enough information that we can identify the observer. Independently, a detailed comparison of the constellation symbols on the Atlas with those from the other surviving ancient material also uniquely points to the same origin for the figures.

The Farnese Atlas ${ }^{1}$ first came to modern attention in the early sixteenth century when it became part of the collection of antiquities in the Farnese Palace in Rome, hence its name. The statue was later transferred to the museum in Naples now called the Museo Archeologico Nazionale di Napoli. It is carved in white marble and depicts the bearded Atlas crouched down on one knee with a cloak over his shoulder and holding the celestial globe on his shoulder with both hands. The globe is $65 \mathrm{~cm}$ in diameter. Its top has a substantial hole knocked into it and this has obliterated the constellations of Ursa Major and Ursa Minor. A total of 41 constellations $^{2}$ are depicted, each drawn in positive relief as the classical figure, with no individual stars shown. Art historians conclude that the statue is a Roman copy from the second century A.D. of a Greek original dating to before the birth of Christ. ${ }^{3}$

What is the date of the observations used for depicting the constellation positions on the Farnese Atlas? A very wide range of plausible answers is possible. First, the Roman sculptor could have updated the constellation positions with his own observations (or those of a contemporary), hence suggesting a date of $c .150$ A.D. Second, the Roman sculptor could have used the latest star catalogue to place the constellations accurately onto the coordinate grid of the sky, and this would be from the Almagest of Ptolemy, 


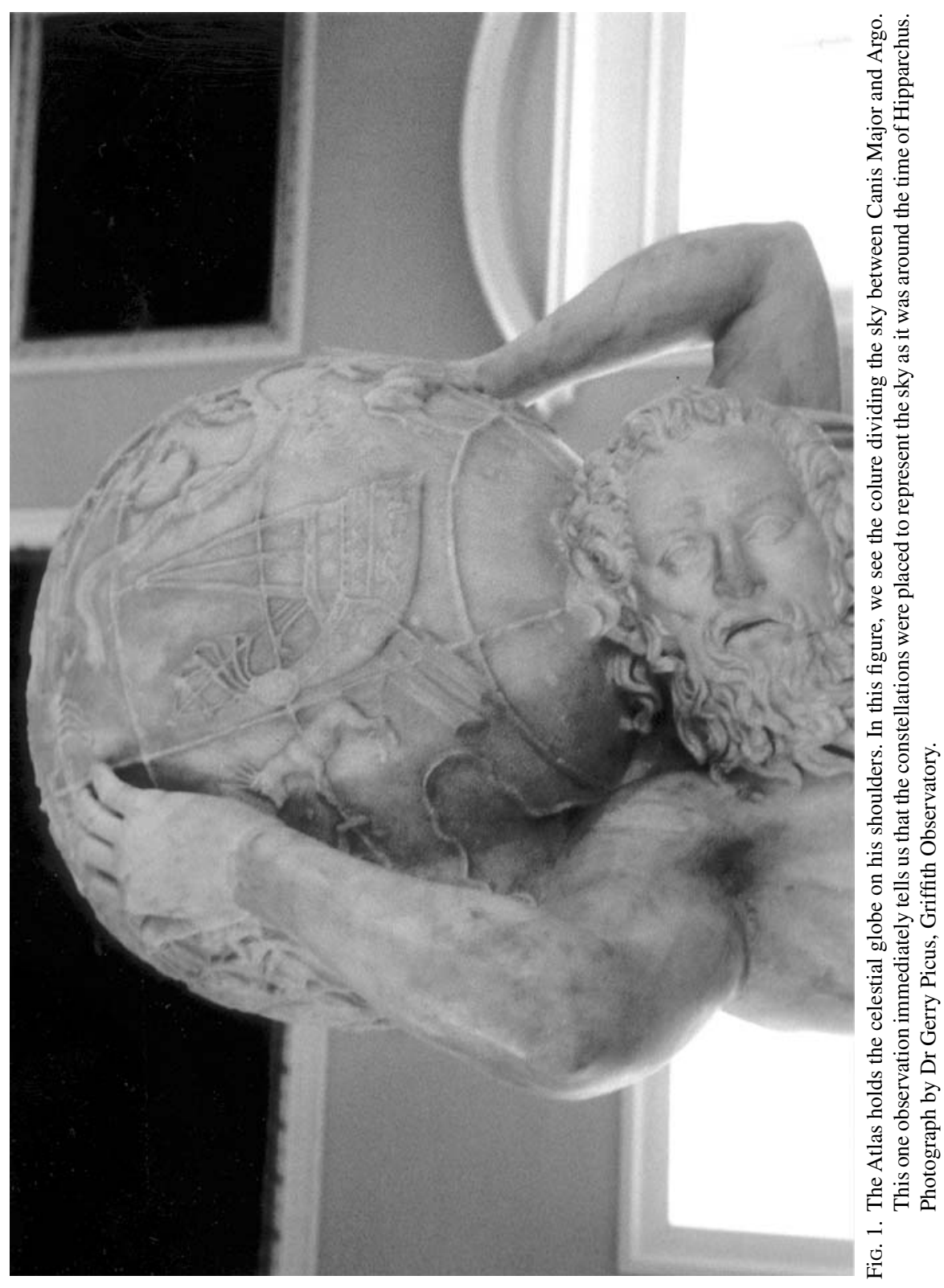




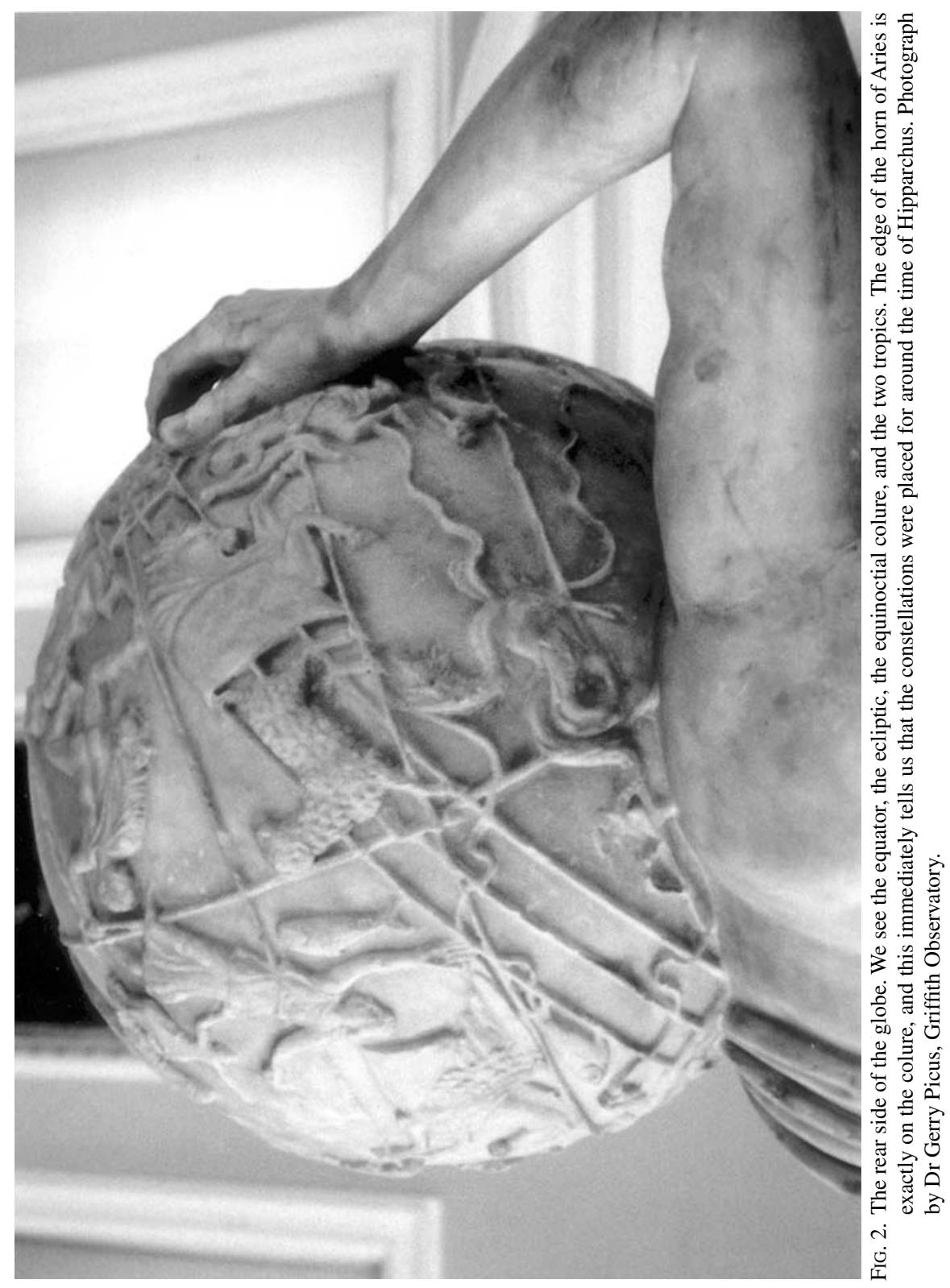


suggesting a date of $c .128$ A.D. Third, art historians all point to the original Greek sculptor as using the constellations based in Aratus's poem Phaenomena, which has a date of $c .275$ B.C. An origin with Aratus was the dominant opinion amongst scholarly publications in the last century. Fourth, we know that Aratus's work was substantially a copy of an earlier book of the same name by Eudoxus with a date of $c .366$ B.C. Fifth, a precessional dating of 172 lore items derived from Eudoxus's book proves that all of the lore actually dates from $1130 \pm 80$ B.C. ${ }^{4}$ So we are left with many candidates, all reasonable, for the date of the observations used to place the constellations: from 1130 B.C. to A.D. 150.

The possibility of deriving a date and latitude from the Farnese Atlas has not been lost on earlier researchers. E. L. Stevenson claims (purely on the basis of the positions of the solstices) that the constellations date "at least three hundred years before the Christian era", while C. Gialanella and V. Valerio as well as M. Fiorini agree that the constellation positions suggest a date in the fourth century B.C., although Valerio later changed the date to A.D. 150. Thiele points to an origin by Hipparchus and Eudoxus based on stylistic considerations, and he also points to a latitude of $23^{\circ}$ which he specifies as being greatly different from that of Rhodes. For the latitude of the observer, Fiorini gives $40^{\circ}$ and points to Macedonia, Gialanella and Valerio give $32^{\circ}$ and point to Alexandria, while Valerio later gives $33.5^{\circ}$ and points to Middle Phoenicia. For the obliquity of the ecliptic, we hear values of $23^{\circ}$ from Fiorini, $25.5^{\circ}$ from Gialanella and Valerio, $25^{\circ}$ from Valerio, and $24^{\circ}$ from G. Aujac. Throughout all the few sentences of discussions by the various authors, no evidence is ever presented, nor are methods of measurement ever discussed, nor is any indication of the accuracy of the claims ever made. With the total lack of these crucial details, we cannot take these off-hand claims seriously. In her appraisal of this situation, Aujac concludes: "A critical review of existing studies of the globe, together with detailed reproduction and careful analysis, is urgently needed to resolve these questions."

This paper will provide the requested analysis. I start with a detailed analysis of the constellation symbols and descriptions on the Farnese Atlas as compared to all relevant surviving sources from Antiquity. In addition, I have taken detailed photographs of the Farnese Atlas under conditions for which photogrammetry can be performed. From these photographs, I have measured the positions of the constellations in the coordinate system of the globe. I have then performed a chi-square analysis to determine the best date (as well as the uncertainties in this value) for the constellation positions. In all, my results will point to the source of the observations with high confidence.

The plan of this paper is to start in Section 2 by making a detailed comparison of the symbols on the Farnese Atlas with those from all other ancient sources. This analysis will include detailed comparison of descriptions of each constellation as compared with the works of Aratus (from the Phaenomena, and hence also Eudoxus), Hipparchus (from his Commentary on the Phaenomena of Aratus and Eudoxus), Ptolemy (from the Almagest), and Psuedo-Eratosthenes (from the Catasterismi and hence also Eratosthenes). In Section 3, I will present the results of my extensive 
photogrammetry, such that I will derive a very confident date for the original observations used to place the constellations onto the globe. This section will also give the declination of the Arctic and Antarctic Circles on the globe and discuss the implications for the latitude of the observer. A third part of Section 3 will discuss the accuracy of the placement of the constellations and the implications for the source of the original observations. Section 4 will put all the results together and a strong case will be made for the identification of the original observer. Section 5 will discuss some implications and applications of this conclusion. Section 6 will summarize the conclusion. This paper has an extensive Appendix, into which I have placed all the technical details relating to the photogrammetry. The goal is to provide complete details so that readers can test my results or perform their own analyses.

\section{DETAILED COMPARISON OF CONSTELLATION FIGURES WITH ANCIENT SOURCES}

The Atlas's globe contains a wealth of information in the form of constellation figures. From a detailed comparison of these symbols with all known sources describing the ancient constellations, we might be able to select and/or eliminate possible sources for the sculptor. Here, I will make this comparison with the works of Aratus (and hence Eudoxus), Hipparchus, Pseudo-Eratosthenes (and presumably Eratosthenes), and Ptolemy.

As a preview, I will highlight several of the more distinct differences. First, the Farnese Atlas is completely missing the later Greek constellations of Equuleus, Coma Berenices, and Antinous. Second, Hercules is depicted as a kneeling man with no clothes and no objects in his hand instead of as the Greek hero. These two facts suggest that the source of the constellations was not in later Greek times. Third, the modern constellation of Libra is depicted as a separate balance, even though the claws of Scorpius extend up to the balance. Fourth, the summer solstice is depicted as being at the start of Cancer near the head of Pollux, in stark contrast to the tradition from Aratus and Eudoxus that the solstice is near the start of Leo. These last two items suggest that the source is after the time of Aratus.

Before I perform the detailed comparison with the individual books, I will give a list of the details that are different from all ancient sources. These details then cannot be useful evidence against any one source, but rather point to changes incorporated after the data left their source (e.g., by the sculptor). Here are the universal differences: (1-2) The horn of the Bull does not touch the foot of the Charioteer, and the head of Andromeda does not overlay the navel of Pegasus, at odds with all ancient descriptions of these constellations. Likely, the sculptor avoided the overlap simply for artistic reasons and clarity. (3) The curious rectangular feature above Cancer corresponds to nothing recorded in any of the ancient sources, and is undoubtedly a later addition by either the Greek or Roman sculptor. (4-5) The globe does not depict Sagitta or Triangulum, whereas every ancient source explicitly discusses both. These are inconspicuous constellations in crowded areas, so perhaps their absence is just an artistic decision by the sculptor. (6) The globe does not depict Ursa Minor, 
although this is attested by all ancient sources. But the Little Bear should be on the very edge of the hole in the top of the globe, so there is a small chance that the figure was in the hole and that there is some other error relating to the positioning near the north pole ( $c f$. Section A.2.2). (7) The ecliptic crosses the equator $5^{\circ}$ west of the colure lines. This arrangement is wrong by definition, as precession moves the sky along the ecliptic, suggesting that the sculptor made the change because of his lack of astronomical knowledge. (8) The string attached to the northern fish of Pisces is missing, although the string is present in all ancient sources. (9) Sagittarius appears to have a bare back, even though the Almagest and Commentaries talk about the cloak-strap. Since all nine differences are universal, they cannot be used to point towards or away from any one source. Instead, these differences indicate changes made after the information had left the astronomer, likely by the sculptor. Similarly, the universal similarities between the globe and all ancient sources (e.g., that only the stern of Argo is depicted) cannot be used to distinguish the origin of the figures. Thus, the only data relevant for determining the origin of the figures are the differences between the globe and sources, which vary with the source.

The Phaenomena of Aratus was a popular description of the constellations; it dates to around 275 B.C, and is the earliest surviving discussion of the ancient Greek constellations. ${ }^{5}$ Its popularity served to freeze the development of the constellations as well as to define the basic properties of the group. The text is largely a version of an earlier (now lost) book of the same name by Eudoxus from around 366 B.C., with substantial further fragments from the work by Eudoxus appearing in the only surviving work of Hipparchus (his Commentary). The Phaenomena gives descriptions of the constellation figures and also tells how these figures relate to the various circles on the sky. A detailed comparison with the Farnese Atlas shows many differences: (1) Aratus stated the summer solstice to be at the start of Leo, whereas the statue shows it to be near the head of Pollux with the entire constellation of Cancer between. (2) The human part of the Centaur is said to be under Scorpius, whereas the statue places it under Virgo, with the entire constellation of Libra between. (3) Eridanus is said to stop at the neck of Cetus while the stars under Lepus are said to be nameless, whereas the Farnese Atlas shows Eridanus as extending all the way through Columba over to the feet of Canis Major. (4) Aratus says that Auriga has the Goat and Kids, whereas the globe in Naples shows the Charioteer instead to be holding a whip. (5) Serpens is said to encircle the waist of Ophiucus, contrary to what is seen on the statue. (6) The constellation of Libra is depicted as a balance on the globe, but Aratus simply calls it "The Claws". (7) The knees of the Charioteer are said to be on the Tropic of Cancer, whereas the globe shows even his feet to be north of the tropic. (8) The left shoulder and shin of Perseus are said to be on the Tropic of Cancer, whereas on the statue all of Perseus is north of the tropic. (9) The head and neck of Cygnus are said to be on the Tropic of Cancer, whereas the sculptor depicts the constellation with the beak tip touching the tropic. (10) Ophiucus's shoulders are said to be along the tropic, whereas the globe shows the top of his head to be there. (11) The knees of Ophiucus are not on the equator as in Aratus, but are depicted as being substantially 
south of the equator. (12) The belt of Cepheus is said by Aratus to be on the Arctic Circle, whereas the Atlas has the neck of Cepheus so drawn. There are many further discrepancies for which the case is less clear, for example Aratus says that the head of Draco is on the Arctic Circle and that Crater and Corvus are on the equator, while the globe shows the head only as being near the Arctic Circle and the raven and cup as only tangent to the equator.

Hipparchus was perhaps the greatest astronomer of Antiquity, his outstanding discovery being the precession of the equinoxes. He is said to have compiled a large star catalogue with at least hundreds of stars, although this catalogue has not survived. Indeed, only one of Hipparchus's works has survived, the Commentary, which gives extensive quotes from both Eudoxus and Aratus. ${ }^{6}$ The thrust of the Commentary is to correct perceived errors in the Phaenomena by quoting the author's own data and interpretation of the constellation figures. As a result, we can make a detailed comparison of Hipparchus's personal descriptions with the Farnese Atlas. First the differences between Hipparchus and the globe: (1) The head of Pollux is close to the Tropic of Cancer (and might even lie upon it), whereas Hipparchus says that the heads of both Twins are north of the Tropic and he even says that Pollux is north of the Tropic by $6^{\circ}$. But that is all: I can find only one non-universal difference between the globe and the Commentary. This difference is only quantitative, and it is only $1.7-\sigma$ in error ( $c f$. Section A.2.1), which is not adequate grounds to claim a significant discrepancy. That is, the small shift of Gemini is typical of the scatter in the placement of the constellations, and so this is not grounds for considering the misplacement to be indicative. Another potential difference is that the globe depicts the modern Libra as a balance with the scorpion's claws extending into it, while Hipparchus usually calls the constellation "The Claws"; however, in one place Hipparchus does recognize the constellation as "The Balance" (Commentary 3.1.5), so this cannot be regarded as being a true difference.

Now let us examine items in which the globe matches details particular to Hipparchus: (A) Hipparchus explicitly corrects Aratus's claim about the position of the Centaur (see item 2 above), and Hipparchus's claim is matched by the depiction on the globe. (B-G) Hipparchus also explicitly corrects Aratus's items 7-12 above, and these corrections are entered onto the globe. (H-J) Hipparchus explicitly corrects the Phaenomena by saying that the head of Draco is only close to the Arctic Circle, and that Crater and Corvus are south of the equator; all these items are as represented on the globe. $(\mathrm{K})$ Hipparchus states that the Arctic Circle is $37^{\circ}$ from the north pole (i.e., at a declination of $53^{\circ}$ ), and this is reasonably consistent with my measured declination of $51.7^{\circ} \pm 0.9^{\circ}$ for the Farnese Atlas. (L) Item 3 (from the penultimate paragraph) is apparently corrected to agreement with the Atlas, as Hipparchus states that Eridanus has a second westward segment. In all, the one difference is statistically insignificant while the dozen agreements between Hipparchus's personal observations and the globe are close, many, and detailed. With this, we see that the comparison between the Atlas and Hipparchus is arguably perfect (other than the 9 itemized differences that are true for all ancient sources). 
The Catasterismi that survives to today is an epitome from $c$. A.D. 100 (by an author known as Pseudo-Eratosthenes) of an original work of the same name by the famous Eratosthenes from $c .245$ B.C. ${ }^{7}$ It is unclear what fraction of the surviving text is from Eratosthenes's composition. The Catasterismi contains a verbal description of the myths and constellations, these being for the most part simply the usual descriptions such as are from Aratus. The Catasterismi does give some non-traditional details that match with the statue, in particular that (A) the Arctic Circle is along the neck of Cepheus, (B) the upheld wrist of Bootes is also along the Arctic Circle, and (C) Eridanus appears above Canopus (hence it must extend through Columba). The Catasterismi differs in many details from those visible on the Farnese Atlas: (1) Auriga is not shown on the Atlas with either the Goat or the Kids. (2) The Asses are not shown on the west side of Cancer. (3) Hercules is not shown on the Atlas as standing. (4) Hercules is not shown as holding a lion skin. (5) Hercules is not shown as holding a club. (6) Pegasus is shown as having wings, despite the explicit denial in the Catasterismi. (7) The constellation Corona Australis is depicted on the globe but never mentioned by the Catasterismi. (9) The modern constellation of Libra is not separated out and is described as simply the claws of Scorpius, unlike what we find on the statue.

Ptolemy's Almagest dates from c. A.D. 128 and contains a long catalogue of stars individually labelled by their positions in the constellations. ${ }^{8}$ These labels allow us to visualize the constellation figure for comparison with the Farnese Atlas. There are many differences between the Almagest and the Atlas: (1) Auriga is not shown on the statue as carrying the Kids. (2-4) The constellations of Equuleus, Coma Berenices, and Antinous are not depicted on the globe. (5) The Almagest identifies the Asses near the middle of Cancer, while these are not displayed on the globe. (6) The modern constellation of Libra is called "The Claws" by the Almagest but is drawn as a balance on the Farnese Atlas. (7) Sagittarius does not have a cloak over the shoulders as stated in the Almagest. (8) In the Almagest Aquarius in not said to have a water jar, although this is clearly depicted on the statue. (9) Canis Major does not have a crown, as shown on the globe, although the crown may simply be a depiction of light rays from Sirius. (10) In the Almagest Eridanus turns south along the modern track near $v$ Eri, rather than extending to near the feet of Canis Major as shown on the Farnese Atlas. (11) Ptolemy explicitly assigns two legs to Cygnus, whereas the Atlas shows only one.

From this detailed analysis, we see that the Farnese Atlas is virtually identical to the constellation description by Hipparchus, yet is greatly different from the descriptions from all other ancient sources. This obviously strongly suggests that the ultimate source of the position information used by the original Greek sculptor was Hipparchus's data, which must be closely related to his (now lost) star catalogue. 


\section{EPOCH AND LATITUDE}

The constellations in the sky move slowly with respect to the declination circles and the colures, as a result of precession. The epoch for the observations that were incorporated into the Farnese Atlas is near that year for which the constellation positions on the globe most closely match those in the sky. The latitude of the observer is related to the declination of the Ant/Arctic Circles as presented on the globe. Both of these calculations require that we get the positions of the constellations in the reference frame of the globe's coordinate system. In principle this could be performed by taking a tape measure to the globe, but in practice an equivalent method is to take pictures of the globe and then carefully measure the pictures.

I took 49 pictures of the Farnese Atlas in Naples on 1 June 2004. I used a digital camera that allowed for good recording of the details without special lighting. For photogrammetry, it is important to know the distance between the camera and the near surface of the globe, as this is required to transform positions on the photograph to spherical coordinates on the globe. (All previous published photographs were taken at unknown distances, and that is why a new set of photographs was required.) My photographs were all taken with the camera at a distance of either 6 or 20 feet from the surface of the globe. There was substantial duplication and some pictures were not useable for various reasons, so I ended up doing photogrammetry on twelve pictures. Detailed explanations and examples for my photogrammetry techniques are presented in Appendix 1. Detailed results and analysis of the constellation positions and the declinations of the tropics and Ant/Arctic Circles are presented in Appendix 2.

\subsection{Epoch}

At a simple level, the date of the Farnese Atlas's astronomical information can be determined by looking at the position of the vernal equinox. For example, Ptolemy gives the position of the westernmost edge of the horns of Aries ( $\gamma$ Ari) as having an ecliptic longitude of $6^{\circ}$ for the year $c$. A.D. 128, while this same star will have a right ascension of zero (i.e., it will lie exactly on the colure) in the year 166 B.C. The Farnese Atlas shows the westernmost edge of the Ram's horn to lie exactly on the colure, so we already know that the constellation position falls some time around the second century B.C. In particular, this one position argues against the Almagest or any late Greek or Roman source for the constellations on the Atlas.

Similarly, we can look at the position of the summer solstice point. According to Aratus (and hence Eudoxus) the summer solstice point is at the beginning of Leo (a statement that was true around 1250 B.C. ${ }^{9}$ ), while the position of the summer solstice is at the beginning of Cancer (just below the head of Pollux) around 1 B.C. The Farnese Atlas shows the summer solstice point to be at the start of Cancer and just beside the head of Pollux. This is a huge and critical difference of an entire constellation in the position of the summer solstice. This argues against any early Greek origin as well as Aratus's or Eudoxus's being the source for the constellations on the globe. 
The two positions discussed in the previous paragraphs are indicative, but the uncertainties associated with any one position are likely to be a few centuries. However, by combining a similar analysis for many points on the Farnese Atlas, these uncertainties can be greatly reduced. The reduction of errors scales as a factor of the inverse square root of the number of points included in the analysis. My analysis in Appendix 2 uses 70 points on the globe, and so my formal (one-sigma) error bar is 55 years. That is, by combining 70 points, I can substantially reduce the various random and happenstance problems, such that I can pin the origin of the Farnese Atlas's constellations to the nearest half-century. This accuracy is good enough for me to be able to specify the historical setting as well as to eliminate all but one of the candidates for the source.

My analysis (see Appendix 2) yields 125 B.c. for the date when some observer made the astronomical observations of the positions of the constellations that ultimately got incorporated into the Farnese Atlas. The one-sigma error bar is \pm 55 years, which means that there is a $68 \%$ probability that the date is between 180 B.C and 70 B.C. The two-sigma error bar gives a $95 \%$ probability that the date is between 245 B.C. and 10 B.C. Within these quoted uncertainties, my result is very strong and solid.

With a very high confidence in the derived epoch of $125 \pm 55$ B.C., we can immediately test the many possible origins. The common conclusion that the constellations are from Aratus (c. 275 в.C.) is greatly inconsistent with this, even if we ignore the fact that the data in Aratus really date to $1130 \pm 80$ B.C. Similarly, the proposal that constellations are from Eudoxus (c. 366 B.C.) or the original Assyrian observer (1130 \pm 80 B.C.) are surely to be rejected. The reasonable possibility that the Roman sculptor (presumably c. A.D. 175 and around $40.9^{\circ}$ latitude) is near the origin of the constellation information is also surely to be rejected. Finally, the possibility that the constellations depicted are based on Ptolemy's Almagest (c. A.D. 128) is eliminated. In all, we have confidently ruled out all published proposals for the origin of the constellation figure data on the Farnese Atlas. The only remaining reasonable possibility is that the Farnese Atlas is based on Hipparchus's star catalogue (c. 129 B.C.). Here we have a perfect match in date with what is seen on the Farnese Atlas. So for the second time, with completely independent reasoning, we confidently eliminate all known possibilities except Hipparchus and we find a perfect match with Hipparchus.

\subsection{Latitude}

Appendix 2 presents an analysis that derives the declination of the Ant/Arctic Circles to be $\pm 51.7^{\circ} \pm 0.9^{\circ}$. This datum must be related in some sense to the latitude of the observer. There are three reasonable interpretations.

The obvious interpretation is that this value is $90^{\circ}$ minus the observer's latitude. This case is where a mathematically-inclined observer measured his own latitude and derived the position of the Ant/Arctic Circles as being that angle from both poles. Thus, the observer (whose report was used ultimately by the sculptor) was at a latitude of $38.3^{\circ} \pm 0.9^{\circ}$. This parallel cuts through the Straits of Messina, Athens, 
and central Turkey. This would likely rule out that the observer was in Mesopotamia $\left(30^{\circ}-36^{\circ}\right)$, Alexandria $\left(31.2^{\circ}\right)$, or near Rome $\left(41.9^{\circ}\right)$. This latitude is consistent with an origin in classical Greece $\left(36^{\circ}-40^{\circ}\right)$ as well as being not greatly inconsistent with Hipparchus in Rhodes $\left(36.4^{\circ}\right)$.

The second interpretation is that the depicted circles might be intended to match actual observations of the lowest declination where the stars never set and of the most southerly limits of visibility. For the Arctic Circle, the observer might have a northern horizon that is higher up than an ideal horizon or he might have adopted a visibility definition such that a star is circumpolar only if it is actually visible at its lower meridian passage. In the latter case, the adopted declination would be closer to the pole and lead to our deriving a latitude that is too far north, and hence the latitude of the observer might be closer to $34^{\circ}$. For the Antarctic Circle, the effects of normal extinction in the atmosphere results in a significant difference between the ideal southern horizon and the actual southernmost visible star depicted. This difference is roughly $4^{\circ}$ for the bright southern stars of relevance..$^{10}$ Hence, the visibility conditions would suggest an observer farther south, perhaps at a latitude of $34^{\circ}$. Other interpretations are possible as an intermediary between the 'obvious' value $\left(38.3^{\circ}\right)$ and the visibility value $\left(\sim 34^{\circ}\right)$. For example, perhaps the placement of the circles on the globes was made by the mathematical calculation based on the known latitude while the actual visible constellation figures were stretched to reach these circles (causing the distortion in declination noted in the Appendix). With this interpretation, the latitude of the observer could be from roughly $34^{\circ}$ to $38^{\circ}$. With this extension, the latitude of Hipparchus in Rhodes $\left(36.4^{\circ}\right)$ becomes easily acceptable.

James Evans has suggested a reasonable third interpretation. He points out that Geminus implies that there was something like a standard latitude for the manufacture of celestial globes when he says "all the spheres are inscribed for the horizon of Greece", and he explicitly remarks in this context that the Arctic Circle is 6/60 of a full circle (i.e., $36^{\circ}$ ) from the pole. ${ }^{11}$ That is, apparently the Arctic Circle on globes are standardized to be at $54^{\circ}$ in declination, although the universality of this convention is not complete. For example, Hipparchus's Commentary gives a value of $53^{\circ}$. The existence of such conventions is common, for example in the placement of $\alpha=0^{\circ}$ at the vernal equinox for some standard epoch even for mapmakers at other epochs, as well as the placement of the prime meridian at Greenwich even for mapmakers far from England. The Farnese Atlas might either have its Arctic Circle slightly misplaced as a standard or have adopted some alternative standard. In such a case, the observer could have been anywhere in the Greco-Roman world.

In all, the declinations of the Ant/Arctic Circles $\left( \pm 51.7^{\circ} \pm 0.9^{\circ}\right)$ has an unknown relation to the latitude of the observer who provided the constellation positions. Any observer in the Greco-Roman world is consistent with this constraint. 


\subsection{Accuracy of the Constellation Placement}

The constellations are placed onto the Farnese Atlas with remarkable accuracy. From Appendix 2, I find that the constellation positions have an accuracy of $3.5^{\circ}$ along the various celestial circles and of $5^{\circ}$ away from those circles. (The difference between on-circle and off-circle accuracy is likely due simply to the sculptor's being less well able to interpolate the positions between the marked grid lines.) Given the many and various factors contributing to this observed accuracy, the original data source must have been substantially more accurate than $3.5^{\circ}$. An estimate of the sizes of the other sources of scatter suggests that the original source must provide the positions at least as accurately as $\sim 2^{\circ}$ or better. This fact can give us an indication of the nature of the data source.

The constellation positions in Aratus (and Eudoxus) are simply verbal descriptions. The accuracy at which they place points along the various declination circles and colures is $4^{\circ} .{ }^{12}$ This is substantially worse than what is required to place the constellations onto the Farnese Atlas. As such, the known verbal descriptions of the constellations are not likely to be the source for the sculptor.

A star catalogue (with measured positions for stars identified as particular parts of the figures) allows for accurate placement of the constellations onto a celestial globe. The typical positional error for stars in the Almagest is rather better than $1^{\circ}$, and the star catalogue of Hipparchus undoubtedly had comparable accuracy. This is fully consistent with the observed accuracy for the Farnese Atlas. The Farnese Atlas will have additional errors added to the star catalogue errors, due to the sculptor (both in his not placing the constellations correctly according to the catalogue in hand and in his drawing the figures in natural poses) and to my measurement errors (resulting from both the usual uncertainties in photogrammetry of $1^{\circ}-2^{\circ}$ and my choice of the exact place in the figure to identify with the star position). In all, the total error in my derived positions for stars on the Farnese Atlas should be $\sim 3^{\circ}$ or worse, if the sculptor based the figures on a star catalogue. In practice, the original Greek sculptor might well have been working from a functional globe made by some astronomer and based on a star catalogue.

Thus, the fine placement of the constellations implies that the original source of astronomical data was a star catalogue. Only two star catalogues are known from the ancient Western world, those of Hipparchus and Ptolemy.

\section{THE FARNESE ATLAS AND HIPPARCHUS'S LOST STAR CATALOGUE}

Let me summarize the main results of what we know about the source for the constellation positions on the Farnese Atlas. First, the constellation symbols and relations are identical with those of Hipparchus and are greatly different from all other known ancient sources. Second, the date of the original observations is $125 \pm 55$ B.C., a range that includes the date of Hipparchus's star catalogue (c. 129 B.C.) but excludes the dates of all other known plausible sources. Third, the accuracy of the original data source must be $\sim 2^{\circ}$ or better, which implies that the source was a star catalogue, and 
the only known star catalogues are those of Hipparchus and Ptolemy. These three strong results all compel us to the conclusion that Hipparchus's lost star catalogue is the source of the constellations on the Farnese Atlas.

Nevertheless, it is prudent to take a further step, to check in every way possible that the conclusion is consistent with everything else we know about ancient Greek astronomy. Many aspects of the claim can be checked for consistency:

(1) Is it plausible to date celestial globes back to the time of Hipparchus? The Almagest (Book VIII, chap. 3) gives a detailed discussion on the construction of solid globes for showing stars. The concept of star globes was common in Greek times, as evidenced by remarks of Geminus (first century A.D.) that assumed widespread familiarity with the concept, by remarks by Cicero that Eudoxus (c. 366 B.C.) and Archimedes (c. 287-212 B.C.) possessed globes, and by the existence of many Greek and Roman coins and engraved gems that show such globes. ${ }^{13}$ In particular, a small bronze coin from Roman Bithynia depicts Hipparchus seated in front of a globe resting on a table. But the primary evidence that star globes date back at least to Hipparchus is that Ptolemy specifically states that Hipparchus had a celestial globe (Almagest, Book VII, chap. 1).

(2) Is the obliquity of the Farnese Atlas consistent with the value used by Hipparchus? From the Almagest (Book I, chap. 12), we are told that Hipparchus adopted an obliquity of $23.85^{\circ}$. As we shall see in the Appendix, I found that the obliquity adopted for the Farnese Atlas was $23.95^{\circ} \pm 0.8^{\circ}$. These two values are consistent.

(3) The latitude of Hipparchus in Rhodes was $36.4^{\circ}$, and this is consistent with all three interpretations for the position of the Ant/Arctic Circles.

(4) The art-historical view of the Farnese Atlas is that it is a copy of a Greek original statue made sometime before around 1 B.C. Presumably, the sculptor made use of some Greek astronomer's observations that were known at the time. Again, this is fully consistent with the source's being Hipparchus.

(5) Likely the original Greek sculptor was not knowledgeable in astronomy, perhaps even to the point of his not being able to use a star catalogue. In this plausible case, the sculptor would need some visual aid, and maybe that aid came as a working celestial globe with the constellations already laid out with respect to the grid of circles. We know that Hipparchus made such globes, so it is quite possible that the Greek sculptor got hold of one of Hipparchus's globes and based the Atlas's globe on this model.

For every point on which we can check, therefore, the Farnese Atlas is found to be consistent with what is known about Hipparchus's lost star catalogue, which strongly supports our conclusion that the Farnese Atlas is indeed based on Hipparchus's catalogue.

The globe on the Farnese Atlas is not a perfect rendition of the Hipparchus star 
catalogue, as there are small random errors in position introduced by the scupltors as well as a variety of universal differences that must have been made after the figures left Hipparchus. There may be substantial uncertainties in taking a figure's position on the globe to be identical to that in Hipparchus's catalogue for purposes of comparison with the Almagest.

\section{IMPLICATIONS AND APPLICATIONS}

As a result of this investigation we can see the skies as observed by the greatest ancient astronomer, and recorded by him in the earliest Western star catalogue. This discovery also sheds light on several major questions that have been debated among historians.

One concerns the type of coordinate system used by Hipparchus. This question has been widely discussed, even in recent years. The conventional view is that "it is quite obvious that at Hipparchus's time a definite system of spherical coordinates for stellar positions did not yet exist". ${ }^{14}$ Nevertheless, some particularly large errors for three partial star positions given in Hipparchus's Commentary can simply be explained as errors that could occur only if Hipparchus was using ecliptic coordinates. ${ }^{15}$ Alternatively, a variety of arguments can be presented in support of the view that Hipparchus used equatorial coordinates, the simplest being that the Commentary reports most of the fragmentary star positions in the equivalent of right ascension and declination. ${ }^{16}$ Duke goes further and points out that the possession of a celestial globe by Hipparchus is possible only if he employed "some sort of 'definite system of spherical coordinates', which Neugebauer assured us 'did not yet exist' at the time of Hipparchus". I believe that the Farnese Atlas will be the key to the continuation of such debates, but I do not know how the arguments will play out. My first reaction is that the globe shows clear circles of constant declination and the colures, and hence is manifestly an equatorial coordinate system. But it could be that the various circles are included merely as part of a tradition for demarcating the sky with the circles mentioned by Aratus and Eudoxus, with no implications for what (if any) coordinate system was used by Hipparchus. (A terrestrial analogy would be that my old hometown has a grid of main streets that are cardinally oriented, but this does not prove that the townfolk use latitude and longitude. A celestial analogy is that modern constellation boundaries are orthogonal for the equinox of 1875 , whereas all working astronomers now use J2000 coordinates.) And Duke's prior argument now has more force, as the existence of accurately placed constellations on a globe (as well as the underlying star catalogue) virtually demands the existence of a coherent spherical coordinate system by the later years of Hipparchus, even if Hipparchus had no single system in his early Commentary. We will have to wait to see what the implications of the Farnese Atlas are for this question.

A second question concerns the relation between Hipparchus's star catalogue and that of the Almagest. This debate has been long and bitter over the centuries, and it has only gotten harsher in the last few decades. ${ }^{17}$ A primary approach has been 
through efforts to make partial reconstructions of Hipparchus's catalogue, based on fragmentary measures discussed in the text of his Commentary. ${ }^{18}$ Now, with the full sky coverage of the Farnese Atlas, we at last have access to all of Hipparchus's star catalogue (and not only to partial positions for a small fraction of the stars). As such, I foresee that the Farnese Atlas will take centre stage in the dispute, as it is the only new source of information for over a century. With this, someone should make a very complete catalogue of all constellation positions on the globe, perhaps involving all positions that correlate with the thousand stars in the Almagest and not merely the 70 positions reported in the appendices of this paper. A substantial disadvantage of this approach will be that the globe positions will be less accurate than the original star catalogue. Nevertheless, I predict that there will be a 'cottage industry' of comparing the Farnese Atlas with the Almagest.

\section{CONCLUSIONS}

This paper provides the first effective examination of the positions of the constellations on the Farnese Atlas. Here are my conclusions:

(1) The epoch for the observations that were used ultimately by the sculptor to place the constellations onto the coordinate grid is $125 \pm 55$ B.C. This is a very strong conclusion, with no real likelihood that this date could simply be the result of historical vagaries or errors (random or systematic).

(2) The declinations of the Arctic and Antarctic Circles are $\pm 51.7^{\circ} \pm 0.9^{\circ}$. There are three reasonable explanations of this value. The obvious one is that the observer was at a latitude of $38.3^{\circ} \pm 0.9^{\circ}$, which is a circle that runs through the Straits of Messina to Athens and to the middle of Turkey. A second explanation is that the intention of the observer or sculptor was to follow the real visibility of the stars, and this allows the observer to be up to $\sim 4^{\circ}$ farther south, i.e., from roughly $34^{\circ}$ to $38^{\circ}$. A third possible explanation is that the sculptor placed the Ant/Arctic Circles to correspond to some 'standard' latitude.

(3) The obliquity of the ecliptic on the globe is $23.95^{\circ} \pm 0.8^{\circ}$. This is easily consistent with the value adopted by both Hipparchus and Ptolemy $\left(23.85^{\circ}\right)$ as well as with the actual obliquity of the time $\left(23.71^{\circ}\right)$.

(4) The positional accuracy for the placement of constellation figures shows that the original source of the data had a positional accuracy of $\sim 2^{\circ}$ or better. This makes it likely that the original observations were recorded as a star catalogue and not as a verbal description.

(5) All previously published proposals for the origin of the observations are easily ruled out with high confidence as a result of the above results.

(6) A detailed comparison of the Farnese Atlas with all surviving ancient sources 
shows a virtually perfect match with the constellation descriptions of Hipparchus. In contrast, all other ancient sources differ profoundly from the Atlas.

(7) The constellations on the Farnese Atlas are based on the now-lost star catalogue of Hipparchus. This is proved by the perfect match with the constellation symbols used by Hipparchus and only for these, by the perfect match with the date of Hipparchus (with the exclusion of all other known candidate sources), by the requirement that the source be a star catalogue such as that compiled by Hipparchus, and by the many points of consistency with what we know about ancient Greek astronomy.

(8) The obvious scenario is that Hipparchus constructed a small working globe based on his (now lost) star catalogue, that this globe was then used by the original Greek sculptor as a model for the constellation placement on a statue, and that the later Roman sculptor used the (now lost) Greek statue to create the globe that is now in Naples.

(9) The existence of this 'new' source for Hipparchus's catalogue is likely to be valuable for our understanding of Hipparchus's astronomical methods and for investigations of the origin of the star catalogue in the Almagest.

\section{APPENDIX 1: PHOTOGRAMMETRY}

Photogrammetry is the process of deriving quantities by the detailed measurement and analysis of photographs. In the case of the Farnese Atlas, I want to be able to measure the declination of the tropics, the declination of the Ant/Arctic circles, and the right ascensions and declinations for many points within the constellation figures. This appendix will present the detailed procedure that I used for my photogrammetry, as well as one worked example.

\section{A.1.1. Angular Distances Between Two Points on the Globe}

When each photograph was taken, I noted the distance between the camera and the surface of the globe $\left(\mathrm{D}_{\text {camera }}\right)$. The physical radius of the globe $\left(\mathrm{R}_{\text {globe }}\right)$ is $32.5 \mathrm{~cm}$. Each picture was printed onto a sheet of paper with a zoom such that the globe filled the page. On the printed picture, the radius of the globe was then measured $\left(\rho_{\text {globe }}\right)$, typical values being $100 \mathrm{~mm}$. The centre of the globe's image was then found either by use of construction techniques straight from simple geometry or by trial and error with a compass. The accuracy of the centre determination was typically $\sim 1 \%$ of the globe radius. The globe is spherical in shape to within $\sim 1 \%$ of the radius, the dominant scatter being caused by the relief depictions of the constellations. (The only exception to this spherical shape is related to the hole gouged in the northern skies which has obliterated Ursa Major and Ursa Minor.) Onto this printed picture, I then drew an orthogonal coordinate system with the origin at the centre of the globe. With this system, every point on the visible surface will have coordinates $\mathrm{X}$ and $\mathrm{Y}$, as measured with a ruler in millimetres from the appropriate axis. The precision of 
my measures is one millimetre.

The first transformation is from this rectangular coordinate system on the photograph $(X, Y)$ to polar coordinates on the photograph $(\rho, \theta)$. The polar coordinates are the distance from the centre, $\rho=\left(\mathrm{X}^{2}+\mathrm{Y}^{2}\right)^{0.5}$, and the angle from the positive $\mathrm{X}$ axis, $\theta=\tan ^{-1}(\mathrm{Y} / \mathrm{X})$.

The second transformation is from the polar coordinate system on the photograph $(\rho, \theta)$ to a spherical coordinate system centred on the camera $(\zeta, \eta)$. The angle $\eta$ is the azimuth angle from the direction of the positive $X$ axis, so that $\eta=\theta$. The angle $\zeta$ is the angle between the sub-camera point on the globe to the point of interest on the globe as viewed from the camera. In this coordinate system, the edge of the globe will satisfy the equation

The angle $\zeta$ can be found from

$$
\sin \zeta_{\text {edge }}=\mathrm{R}_{\text {globe }} /\left(\mathrm{R}_{\text {globe }}+\mathrm{D}_{\text {camera }}\right)
$$

$$
\tan \zeta=\tan \zeta_{\text {edge }} \times\left(\rho / \rho_{\text {globe }}\right)
$$

The third transformation is from this spherical coordinate system $(\zeta, \eta)$ centred on the camera to a spherical coordinate system $(\Phi, \Psi)$ centred on the middle of the globe. The azimuth of the point, $\Phi$, will simply be the same value as $\eta$. As viewed from the centre, the angle between the zenith (sub-camera) point and the point of interest will be $\Psi$. By applying the Law of Sines to the triangle defined by the camera, the centre of the globe, and the point of interest, we find

$$
\sin (\mathrm{A}) /\left(\mathrm{R}_{\text {globe }}+\mathrm{D}_{\text {camera }}\right)=\sin (\zeta) / \mathrm{R}_{\text {globe }},
$$

where $\mathrm{A}$ is the angle subtended between the camera and the globe centre as viewed from the point of interest. In this same triangle, the angle $\Psi$ is simply $180^{\circ}-\zeta-\mathrm{A}$.

We can now convert all the positions measured on the photograph into spherical coordinates for the globe. The next task is to calculate the angular distances $\Gamma$ (within the spherical coordinates) between any two points on the globe. Let the two points have coordinates $\left(\Phi_{1}, \Psi_{1}\right)$ and $\left(\Phi_{2}, \Psi_{2}\right)$. We can define a spherical triangle from the sub-camera point and the two points labelled with subscript ' 1 ' and ' 2 '. From the Law of Cosines for spherical triangles, we find that $\cos \Gamma=\cos \Psi_{1} \times \cos \Psi_{2}+\sin \Psi_{1} \times \sin \Psi_{2} \times \cos \left(\Phi_{1}-\Phi_{2}\right)$.

Thus, we can determine the angle between any two visible points on the globe.

\section{A.1.2. Declinations for Tropics and Ant/Arctic Circles from Photogrammetry}

With this framework, we can now calculate the angular distances between the equator and the other circles along the great circles formed by the colures. For each picture, along each of the colures visible, I placed a dot of coloured ink at the exact crossing point with each of the equator, tropic, and Ant/Arctic circles. I then measured the X and Y rectangular coordinates of each dot. With an EXCEL spreadsheet, the conversion to $\Phi$ and $\Psi$ coordinates was easy. Then, for a given colure, I calculated the angle between the equator and the circles. This procedure yields the declination for each circle as based on that one photograph. For each intersection, I have an average of 3.5 measures of declination. The RMS scatter of these separate measures has a typical value of $0.5^{\circ}$, and this represents my measurement error. These values are averaged 
together to get the best value for the declination of the circle along that colure. The declinations of each circle are all constant to within the rather small uncertainties, and this demonstrates that the sculptor made good parallel circles to within an accuracy of $0.2^{\circ}-0.5^{\circ}$. The RMS scatter in all the measured values is divided by the square root of the number of independent measures to determine the one-sigma uncertainty in the measured declinations.

\section{A.1.3. Right Ascensions and Declinations for Any Position}

The primary task for my photogrammetry is to go from the measured position on the photograph to the right ascension and declination of the star in the reference frame as defined by the grid of circles on the globe. Section A.1.1 of this Appendix tells how to go from measured positions on the photograph to spherical coordinates on the globe with the sub-camera point being the 'pole'. In principle, a suitable triple of rotations in the spherical coordinates will transform to the equatorial coordinate system. Instead, I have adopted an easier method: I (a) choose two widely-spaced cross points of grid circles, (b) calculate the angular distance between the point of interest and both of the reference points using the formula from the first section, (c) adopt some approximate right ascension and declination for the point of interest, (d) calculate the distance between the currently adopted position on the sky and the right ascension and declination of the reference points, (e) compare the observed angular distances from steps (b) and (d), and (f) repeat steps (c)-(e) with successive refinements in the adopted position until the agreement is satisfactorily close. This iterative numerical procedure is fast and accurate.

The reference points are usually taken to be where a colure intersects the two tropic circles. The adopted declinations for these points of intersection must be those of the photogrammetric coordinate grid, so the tropics are taken to be at $\pm 26.2^{\circ}$ while the Ant/Arctic circles are taken to be at $\pm 57.5^{\circ}$. In principle, there will always be two points on the sky that have the same angular distances from the two reference points as on the globe, but this ambiguity is always easy to resolve with certainty on the basis of the visible position on the globe. In this iterative process, "satisfactorily close" is to better than $0.1^{\circ}$ for my calculations. The result will be a position in the photogrammetric coordinate system and must be corrected to the real sky. As discussed in the next appendix, there is a small distortion in declination, such that positions in the photogrammetric coordinate system must have their declination corrected to that of the real sky. This correction is made by subtracting an offset to the magnitude of the declination which is found by a linear interpolation to vary from $0.0^{\circ}$ on the equator to $2.25^{\circ}$ on the tropics and $5.8^{\circ}$ on the Ant/Arctic circles. The result will be the derived right ascension and declination for the object as based on that one photograph. Measures of the position on multiple pictures of the Farnese Atlas will provide largely independent measures of the coordinates, and the averaging together of these positions will help reduce the measurement error. So, finally, the end result is an averaged right ascension and declination of the indicated position on the sky as depicted on the globe. 
TABLE 1. Measured positions for first photograph.

$\begin{array}{lcccccc}\text { Point } & \mathrm{X}(\mathrm{mm}) & \mathrm{Y}(\mathrm{mm}) & \zeta(\mathrm{rad}) & \mathrm{A}(\mathrm{rad}) & \Psi(\mathrm{rad}) & \theta=\eta=\Phi(\mathrm{rad}) \\ \alpha=0^{\circ}, \delta=0^{\circ} & -7 & -14 & 0.023 & 2.987 & 0.131 & -2.03 \\ \alpha=0^{\circ}, \delta=-26.2^{\circ} & 5 & -60 & 0.089 & 2.512 & 0.541 & -1.49 \\ \alpha=0^{\circ}, \delta=26.2^{\circ} & -17 & 38 & 0.062 & 2.721 & 0.359 & 1.99 \\ \alpha=0^{\circ}, \delta=57.5^{\circ} & -25 & 85 & 0.131 & 2.101 & 0.910 & 1.86 \\ \alpha \text { Ari } & -3 & 30 & 0.045 & 2.841 & 0.256 & 1.67 \\ \beta \text { Per } & 28 & 60 & 0.098 & 2.438 & 0.606 & 1.13 \\ \alpha \text { Tau } & 70 & 36 & 0.116 & 2.266 & 0.759 & 0.48 \\ \varepsilon \text { Ori } & 94 & 10 & 0.139 & 1.975 & 1.027 & 0.11 \\ \alpha \text { And } & -52 & 15 & 0.080 & 2.583 & 0.478 & 2.86 \\ \alpha \text { Cas } & -57 & 59 & 0.121 & 2.215 & 0.806 & 2.34 \\ \varepsilon \text { Peg } & -96 & -5 & 0.142 & 1.934 & 1.066 & -3.09 \\ \gamma \text { Psc } & -79 & -33 & 0.126 & 2.155 & 0.860 & -2.75\end{array}$

Many uncertainties contribute to the error bars. First, there is my measurement errors, which are $1^{\circ}-2^{\circ}$ as based on the repeatability of positions as measured from picture-to-picture. Second, there is the uncertainty as to my placement of the dot on the constellation figures. For example, does the star $\alpha$ Her correspond to the top or the middle of the head of Hercules? Third, the sculptor will not have placed the constellation figure perfectly with respect to the position of the star, for example because the sculptor has a high priority in not making the constellation figures look wrongly elongated. Fourth, the original observations on which the sculptor is working will not be perfectly accurate. The star catalogue in the Almagest has positional accuracies of a little better than a degree, whereas the verbal descriptions in Aratus are accurate only to around $4^{\circ}$ for placing parts of constellations onto the celestial circles. Fifth, the Roman sculptor did not make a perfect reproduction of the original Greek statue, and this introduces yet more errors.

\section{A.1.4. A Worked Example}

I will here present a detailed example, with all intermediate values presented. This will allow researchers to test my procedures and to see typical values, and will provide a known example to check later applications. I will take for my example the first of my photographs, which is a typical case with neither large nor small error bars.

The photograph was taken with the camera 6 feet from the edge of the globe $\left(\mathrm{D}_{\text {camera }}\right.$ $=183 \mathrm{~cm}$ ). Recall that $R_{\text {globe }}=32.5 \mathrm{~cm}$. The angular radius of the globe as viewed from the camera has $\zeta_{\text {edge }}=0.151 \mathrm{rad}$. The image of the globe was expanded and printed onto paper such that the radius of the image was $\rho_{\text {edge }}=10.3 \mathrm{~cm}$. The centre of the image was found by repeated trials with a compass until all edges were within $0.1 \mathrm{~cm}$ of a circle drawn around this centre (except for a $45^{\circ}$ arc to the north caused by the hole in the globe). I constructed a rectangular coordinate with an origin at this centre and with the $\mathrm{Y}$ axis roughly towards the north. I next placed red dots where the vernal equinox colure intersected the two tropics and the Arctic Circle. I also placed 


TABLE 2. Derived right ascensions and declinations for first photograph.
$\begin{array}{lccccc}\text { Point } & \Gamma * 1^{\circ}\left(^{\circ}\right) & \Gamma * 2\left(^{\circ}\right) & \alpha\left(^{\circ}\right) & \delta_{\operatorname{dist}}\left(^{\circ}\right) & \delta\left(^{\circ}\right) \\ \alpha \text { Ari } & 45.6 & 8.0 & -4.2 & 19.2 & 17.6 \\ \beta \text { Per } & 63.3 & 25.8 & 29.0 & 30.6 & 27.9 \\ \alpha \text { Tau } & 60.9 & 46.2 & 47.5 & 13.5 & 12.3 \\ \varepsilon \text { Ori } & 64.3 & 67.0 & 62.6 & -2.7 & -2.5 \\ \alpha \text { And } & 47.4 & 20.7 & 340.0 & 17.0 & 15.5 \\ \alpha \text { Cas } & 72.2 & 27.6 & 331.9 & 41.1 & 37.2 \\ \varepsilon \text { Peg } & 66.4 & 55.7 & 303.2 & 10.6 & 9.7 \\ \gamma \text { Psc } & 47.2 & 51.9 & 316.4 & -4.0 & -3.7\end{array}$

green dots at 8 positions in constellations that can be identified with distinct stars in the modern sky. Then, with a millimetre ruler, I measured the distance of each dot from the two axes (see Table 1). With these positions, I then made calculations (in EXCEL) as presented in Appendix A.1.1 so as to convert to spherical coordinates on the globe. Intermediary and final values are presented in Table 1.

The angular distances from the equator, along the colure, for the points on the Tropic of Capricorn, the Tropic of Cancer, and the Arctic Circle are then $24.8^{\circ}$, $25.9^{\circ}$, and $57.8^{\circ}$ respectively. These numbers are averaged together with similar numbers from other pictures and along other colures so as to get the values reported in Section A.1.2.

I have adopted two points to define the globe's coordinate system. These reference points are where the equinoctial colure crosses the two tropics, and these points were chosen as they give a long baseline which does not get near to the edge. For each of the eight constellation positions, I then calculate the angular distances from the star to both reference points $\left(\Gamma_{*_{1}}\right.$ and $\left.\Gamma_{*_{2}}\right)$ in the $(\Phi, \Psi)$ coordinate system using the formula near the end of Section A.1.1. By trial and error (which can be done fast within EXCEL), I then find a position on the sky whose right ascension $(\alpha)$ and declination $\left(\delta_{\text {dist }}\right)$, for the distorted coordinates of the globe has identical distances from the two reference points (assumed to be at $\alpha=0^{\circ}$ and $\delta= \pm 26.2^{\circ}$ in the globe coordinate system). These distances and globe positions are presented in Table 2. The final step is to correct the distorted declination to the correct declination for the sky by means of a linear interpolation. This linear interpolation has a subtractive correction of $0^{\circ}$ on the equator, $2.25^{\circ}$ at $\delta_{\text {dist }}= \pm 26.2^{\circ}$, and $5.8^{\circ}$ at $\delta_{\text {dist }}= \pm 57.5^{\circ}$. The final declination is presented in the last column of Table 2 . The end result is measured $\alpha$ and $\delta$ for all eight positions on this one photograph. These values will be averaged with corresponding measures for the same point as made on separate photographs, with the final coordinates presented in Table 5 .

\section{APPENDIX 2: RESULTS FROM POSITIONAL ANALYSIS}

The positions of the constellations on the globe carry information about the date of the observations ultimately used by the sculptor. In addition, the declinations of the tropic and Ant/Arctic Circles will give us the information about the adopted obliquity 
TABLE 3. Positions of stars on circles.

\begin{tabular}{|c|c|c|c|c|c|c|}
\hline$\#$ & Circle & Position Description & Star & $\alpha_{-125}\left(^{\circ}\right)$ & $\delta_{-125}\left({ }^{\circ}\right)$ & $\operatorname{Dev}\left({ }^{\circ}\right)$ \\
\hline 1 & $\alpha=0^{\circ}$ & Westernmost Aries's horn & $\gamma$ Ari & 0.5 & 7.9 & 0.5 \\
\hline 2 & $\alpha=0^{\circ}$ & Westernmost star in Perseus & $\theta$ Per & 9.1 & 38.5 & 9.1 \\
\hline 3 & $\alpha=0^{\circ}$ & Andromeda left foot & $\gamma$ And & 1.4 & 31.0 & 1.4 \\
\hline 4 & $\alpha=90^{\circ}$ & Tail of Dog & $\eta \mathrm{CMa}$ & 90.1 & -27.1 & 0.1 \\
\hline 5 & $\alpha=90^{\circ}$ & Westernmost shield on stern & p Pup & 92.6 & -25.7 & 2.6 \\
\hline 6 & $\alpha=90^{\circ}$ & Just east of Pollux & $\phi \mathrm{Gem}$ & 85.1 & 29.2 & -4.9 \\
\hline 7 & $\alpha=180^{\circ}$ & Centaur's chest front & $\theta$ Cen & 182.5 & -25.1 & 2.5 \\
\hline 8 & $\alpha=180^{\circ}$ & West edge of Libra & $\lambda$ Vir & 187.0 & -2.4 & 7.0 \\
\hline 9 & $\alpha=180^{\circ}$ & Bootes's western toes & $\tau$ Boo & 180.9 & 28.9 & 0.9 \\
\hline 10 & $\alpha=270^{\circ}$ & Just west of Capricorn's head & $\xi$ Cap & 273.1 & -16.3 & 3.1 \\
\hline 11 & $\alpha=270^{\circ}$ & Just east of Lyra's edge & $\eta \mathrm{Lyr}$ & 270.5 & 37.2 & 0.5 \\
\hline 12 & $\alpha=270^{\circ}$ & Just west of Cygnus's beak & 2 Cyg & 270.2 & 27.5 & 0.2 \\
\hline 13 & Equator & Aries hoof, above Cetus's head & $\sigma$ Ari & 14.7 & 4.8 & 4.8 \\
\hline 14 & Equator & Taurus's right hoof & $v$ Tau & 33.3 & -2.1 & -2.1 \\
\hline 15 & Equator & Middle of Orion's waist & $\delta$ Ori & 56.3 & -4.4 & -4.4 \\
\hline 16 & Equator & Top of Cup & $\theta \mathrm{Crt}$ & 147.3 & 1.3 & 1.3 \\
\hline 17 & Equator & Between Crow and Virgin & $21 \mathrm{Vir}$ & 161.4 & 2.3 & 2.3 \\
\hline 18 & Equator & Ophiucus's right hand & $\vee \mathrm{Oph}$ & 240.9 & -6.8 & -6.8 \\
\hline 19 & Equator & Top of Aquarius's head & $25 \mathrm{Aqr}$ & 297.5 & -5.5 & -5.5 \\
\hline 20 & Equator & Top of Pegasus's head & 35 Peg & 309.8 & -4.8 & -4.8 \\
\hline 21 & Equator & Between Pegasus and Fish & $55 \mathrm{Peg}$ & 320.0 & -1.1 & -1.1 \\
\hline 22 & Cancer & Perseus's foot & $\zeta$ Per & 27.2 & 23.2 & -0.6 \\
\hline 23 & Cancer & Taurus's shoulder & $\psi$ Tau & 30.6 & 20.8 & -3.0 \\
\hline 24 & Cancer & Bottom of Pollux's head & $\phi \mathrm{Gem}$ & 85.1 & 29.2 & 5.3 \\
\hline 25 & Cancer & Middle of Crab & $35 \mathrm{Cnc}$ & 108.4 & 26.4 & 2.5 \\
\hline 26 & Cancer & Lion's chest & $\alpha$ Leo & 122.6 & 20.6 & -3.2 \\
\hline 27 & Cancer & Bootes's left foot bottom & v Boo & 181.4 & 27.2 & 3.4 \\
\hline 28 & Cancer & Bootes's right foot bottom & $\zeta$ Boo & 195.0 & 24.2 & 0.3 \\
\hline 29 & Cancer & Hercules's head & $\alpha \mathrm{Her}$ & 234.8 & 19.1 & -4.8 \\
\hline 30 & Cancer & Beak tip of Cygnus & $\beta$ Cyg & 271.4 & 25.5 & 1.6 \\
\hline 31 & Capricorn & Bottom of Lepus & $\varepsilon$ Lep & 54.2 & -27.3 & -3.5 \\
\hline 32 & Capricorn & Dog's front forefoot & $\beta \mathrm{CMa}$ & 72.5 & -19.2 & 4.7 \\
\hline 33 & Capricorn & Argo's shield middle & 1 Pup & 94.5 & -25.3 & -1.5 \\
\hline 34 & Capricorn & Centaur's shoulder & $\theta$ Cen & 182.5 & -25.1 & -1.3 \\
\hline 35 & Capricorn & Bottom of Scorpion's body & $\tau$ Sco & 217.5 & -21.1 & 2.7 \\
\hline 36 & Capricorn & Top of Sagittarius's bow & $\lambda \mathrm{Sgr}$ & 244.5 & -23.5 & 0.3 \\
\hline 37 & Capricorn & Sagittarius's shoulder & $\sigma \mathrm{Sgr}$ & 250.9 & -25.7 & -1.9 \\
\hline 38 & Arctic & Top of Perseus & $\chi$ Per & 2.5 & 46.0 & -5.7 \\
\hline 39 & Arctic & Auriga's head top & $\tilde{\xi}$ Aur & 46.8 & 51.2 & -0.5 \\
\hline 40 & Arctic & Bootes's head top & $\beta$ Boo & 205.2 & 50.0 & -1.7 \\
\hline 41 & Arctic & Hercules's western knee & $\tau$ Her & 229.4 & 52.7 & 1.0 \\
\hline 42 & Arctic & Hercules's eastern foot & $82 \mathrm{Her}$ & 250.7 & 51.2 & -0.5 \\
\hline 43 & Arctic & Cepheus's chest & $\xi$ Cep & 316.0 & 55.1 & 3.4 \\
\hline 44 & Arctic & Cassiopeia's foot & $1 \mathrm{Cas}$ & 2.6 & 56.3 & 4.6 \\
\hline 45 & Antarctic & Lower rudder end & $\alpha \mathrm{Car}$ & 84.4 & -52.7 & -1.0 \\
\hline 46 & Antarctic & Centaur's rear hoof & $\alpha \mathrm{Cru}$ & 161.4 & -51.4 & 0.3 \\
\hline 47 & Antarctic & Centaur's front hoof & $\alpha$ Cen & 186.1 & -50.0 & 1.7 \\
\hline
\end{tabular}

and latitude. To obtain these results, I have used four procedures based on the photographs. The first procedure is to identify singular points of the constellation figures that fall exactly on the various circles inscribed on the globe. The second procedure 
is to use photogrammetry to measure the declinations of the tropics and Ant/Arctic Circles. This work reveals a small distortion in declination by the sculptor that must be corrected to derive positions on the sky. The third procedure is to use photogrammetry to measure the positions of many individual points within the constellation figures. The fourth procedure is to take all the positions from the globe and fit them to the real sky as a function of the year by means of a standard chi-square analysis, with the result being a best fit year and a quantitative estimate of the uncertainty.

\section{A.2.1. Stars Along Circles}

Direct examination of the photographs of the Farnese Atlas can show what specific parts of various constellations are depicted as being exactly on the globe's circles. For example, the westernmost edge of Aries's horn is drawn as being on the equinoctial colure, and this is unambiguously identified with the star $\gamma$ Ari. The chest of Leo is on the Tropic of Cancer, and this is identified with the star $\alpha$ Leo (Regulus). Lists of these stars can be used to determine the epoch of the Farnese Atlas's constellations as being the year in which these stars-on-circles most closely match the circles. When calculated for this best epoch, the average positions of these stars-on-circles will provide an accurate measure of the positions of the circles.

I have compiled a list of specific stars that correspond to specific positions within constellations depicted as being exactly on one of the celestial circles. This list is presented in Table 3. The individual columns are (1) a running number for counting and reference, (2) the identification of the circle, (3) the position description of the part of the constellation that is exactly on the circle, (4) the modern name for the star that matches the position description, (5) the right ascension $(\alpha)$ in degrees of the indicated star for the epoch 125 B.C., (6) the declination $(\delta)$ in degrees of the indicated star for the epoch 125 B.C., and (7) the deviation in degrees between the target and the observed value. This deviation will depend on the circle; for example item number 1 is the colure for which $\alpha=0^{\circ}$ is the target while the observed value is $\alpha=0.5^{\circ}$ for a deviation in observed-minus-predicted equalling $0.5^{\circ}$. Another example is item number 31 , where the target is $\delta=-23.7^{\circ 19}$ and the star had a declination of $-27.3^{\circ}$ for a deviation of $\left(-27.3^{\circ}\right)-\left(-23.7^{\circ}\right)=-3.6^{\circ}$. The precession was calculated with the exact formula given by J. Meeus. ${ }^{20}$ The choice of the date (125 B.C.) is justified

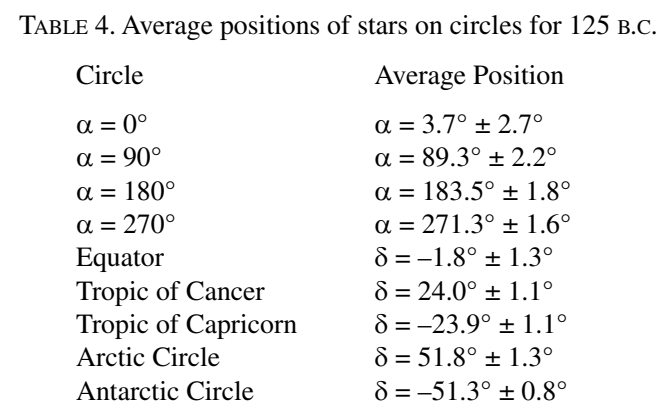


in Sections 3.1 and A.2.4 as being the best fit date.

The RMS scatter of the deviations will provide a measure of the accuracy of the original observations plus whatever later errors accumulate. For each target circle, the RMS scatter varies somewhat about an average of $3.5^{\circ}$. For the observations along the colure, the deviations must be corrected by a factor of $\cos (\delta)$ to account for the convergence of meridians towards the poles. The deviations in Table 3 are scattered with a Gaussian distribution, as 34/47 $=72 \%$ are within one-sigma and $2 / 47=4 \%$ are farther than two-sigma. These average deviations $\left(3.5^{\circ}\right.$ for targets of declination circles and $3.5 \% \cos (\delta)$ for targets of colures) will arise from many causes in addition to the usual uncertainties in the original data. There will be scatter added in when the original data were transferred to a format (likely a working astronomical globe) useable by the original Greek sculptor. And the sculptor will, for purely artistic reasons, have had to shift the figures slightly so that their parts do not appear distorted on the globe (even though the figure on the sky might have some distortions). Then the Roman sculptor will not have made a perfect copy of the original Greek statue. More scatter might have been introduced because the points that I chose on the constellation figures might not have been the positions that corresponded to the modern stars.

Table 4 presents the average coordinates (either right ascension or declination, depending on the circle) for all stars along the given circle as derived from Table 3. The uncertainties quoted on the average equal the RMS scatter divided by the square root of the number of measures. The average positions for the colures will vary systematically with date, increasing roughly by $1.3^{\circ}$ per century. The average positions for the circles of constant declination will vary by only a small amount, one that is substantially smaller than the quoted error bars. For example, the average declination of the stars on the equator and those on the Tropic of Cancer changes respectively from $-1.0^{\circ} \pm 1.5^{\circ}$ to $-1.7^{\circ} \pm 1.3^{\circ}$ and from $23.4^{\circ} \pm 1.3^{\circ}$ to $23.9^{\circ} \pm 1.1^{\circ}$, in the period from 500 B.C. to A.D. 1. So, not surprisingly, the circle declinations are not sensitive to the date and hence it is sufficient to use the average declinations for the best fit date (125 B.C., see Sections 3.1 and A.2.4). We are now in a position to get preliminary answers concerning the Farnese Atlas.

An estimated date for these stars-on-circles can come from looking for the minimum average deviations for the stars along the colures. The average deviation is zero for the year 280 B.C. However, such a criterion is crude since it does not allow for compression of meridian lines far from the equator, nor does it use the optimal chi-square weighting. In addition, there are substantial amounts of further data (see Section A.2.3) which should be combined to derive a final answer. In all, the conclusion about the date of the Farnese Atlas is discussed in Sections 3.1 and A.2.4.

The obliquity of the ecliptic is not sensitive to the epoch, so we can derive it here. The declinations of the two tropics are essentially identical (as expected) and thus we can average together the results of both tropics. From this, I infer that the Farnese Atlas was made for an obliquity of $23.95^{\circ} \pm 0.8^{\circ}$. This is to be compared to the obliquity adopted by both Ptolemy and Hipparchus of $23.85^{\circ}$, as well as with 
the true value of $23.71^{\circ}$. Note that this derived obliquity is obtained by comparing positions of the tropic circles with respect to the constellations, and this is independent of any distortions that may have been built into the coordinate grid of the globe by the sculptor.

The declinations of the Arctic and Antarctic Circles are identical to within the error bars. The combined average of the equatorial distance $\left(51.7^{\circ} \pm 0.9^{\circ}\right)$, and this appears to be the placement intended by the sculptor.

\section{A.2.2. Obliquity and Latitude}

The obliquity of the ecliptic can be measured by photogrammetry as the average declination of the tropic circles. The latitude of the observer will be related to the average declinations of the Arctic and Antarctic Circles as measured by photogrammetry. Appendix A.1.2 presents full details on my procedures for measuring the declinations of the circles on the Farnese Atlas.

I find that the tropics and Ant/Arctic circles are parallel to the equator to within an accuracy of one degree. By averaging together all my photogrammetric measures of the circles' declinations I get the best measures of their declinations. Thus, the Arctic Circle is at a declination of $+57.8^{\circ} \pm 0.5^{\circ}$, the Tropic of Cancer is at a declination of $+26.3^{\circ} \pm 0.2^{\circ}$, the Tropic of Capricorn is at a declination of $-26.0^{\circ} \pm 0.4^{\circ}$, and the Antarctic Circle is at a declination of $-57.0^{\circ} \pm 0.5^{\circ}$.

The equatorial distances of the tropics should equal the obliquity adopted by the Farnese Atlas. The two tropics are at virtually identical distances from the equator, so it is reasonable to form a weighted average and as a result we get an obliquity of $26.2^{\circ} \pm 0.2^{\circ}$. Similarly, we can combine the Arctic and Antarctic Circles to get an equatorial distance of $57.5^{\circ} \pm 0.4^{\circ}$.

These photogrammetry values are substantially different from those based on the circle positions with respect to the background constellations. In fact, the photogrammetry values are larger by $2.25^{\circ} \pm 0.8^{\circ}$ for the tropics $\left(26.2^{\circ}\right.$ versus $\left.23.95^{\circ}\right)$ and larger by $5.8^{\circ} \pm 1.0^{\circ}$ for the Ant/Arctic Circles $\left(57.5^{\circ}\right.$ versus $\left.51.7^{\circ}\right)$. These differences are too large to be by random chance or measurement error. An offset of one degree in declination corresponds to a misplacement of $0.57 \mathrm{~cm}$ on the surface of the globe, so the tropics are off by $1.3 \mathrm{~cm}$ while the circles of invisibility are off by $3.3 \mathrm{~cm}$.

How can we reconcile these differences between the declinations from the stars-oncircles method and the declinations produced by photogrammetry? It is implausible that the sculptor correctly placed the circles on the globes and then systematically misplaced the constellations with respect to the primary coordinate grid on the globe. Also, there is no precedent for there ever being an intentional placement of the tropics for an obliquity of $26.2^{\circ} \pm 0.2^{\circ}$. However, there is excellent precedent for an obliquity of $23.95^{\circ} \pm 0.8^{\circ}$, and this argues that the sculptor was intending to place the tropics and the constellations simultaneously. So the obvious interpretation is that the sculptor placed the declination circles onto the globe with a small distortion that increases with distance from the equator, and then placed the constellations 
accurately with respect to the coordinate grid. Such a distortion in the placement of the grid circles could arise either from an unintentional error on the part of the sculptor or from an intentional decision on his part to improve the display of the constellations for artistic reasons.

The distortion of the globe's coordinates is apparently independent of right ascension (because the tropics and Ant/Arctic Circles are parallel to the equator to an accuracy better than it would be if, for example, the distortion were in ecliptic latitude). The distortion is symmetric north/south. As for the magnitude of the distortion, we only have two values: $2.25^{\circ}$ for the tropics and $5.8^{\circ}$ for the Ant/Arctic Circles. Some model of the distortion is required for photogrammetry of positions away from the circles. While the distortion might well be smooth, many correction functions are plausible, given that we know the curve at only two points. I have therefore adopted the simple piecewise linear function, and this surely produces an additional uncertainty that is small compared to other sources of error.

These two ways of measuring the declination of the circles also accounts for a large disagreement between authorities on the derived obliquity and latitude. Gialanella and Valerio place the tropics at $25.5^{\circ}$ and the Arctic Circle at $58^{\circ}$, so it is clear that they used photogrammetry. Fiorini gives an obliquity of $23^{\circ}$ and a declination of the Ant/Arctic Circles of $50^{\circ}$, so it is clear that he was deriving the declinations of the circles from their positions with respect to the constellation figures.

In all, I conclude that the obliquity used in constructing the Farnese Atlas was $23.95^{\circ} \pm 0.8^{\circ}$ while the Ant/Arctic Circles were $51.7^{\circ} \pm 0.9^{\circ}$ from the equator. The globe has a small distortion in the declination which increases with equatorial distance, and this must be accounted for by the photogrammetry.

\section{A.2.3. Stars Off Circles}

The placement of constellation figures away from the various circles also contains information on the epoch of the globe. That is, a greater or lesser ecliptic longitude on the globe will correspond to a later or an earlier date for the observer. The previous analyses in this paper have not used this source of information, and this task is reported in this section. The idea is to use photogrammetry to derive the right ascension and declination of points within constellations that are readily identified with specific stars, and to convert these positions to ecliptic coordinates. The resultant ecliptic longitudes can then be part of a final fit to derive the approximate year of the observations incorporated into the Farnese Atlas by the sculptor.

Complete details of my photogrammetry are presented in Appendix A.1, along with a worked example for one of my pictures. This provides a mechanism to go from my pictures of the Atlas to positions on the globe.

I have determined 23 specific points within constellation figures that can be unambiguously identified with single stars in the sky. For example, Perseus is depicted as holding a head on his western side near the south (i.e., the Medusa) and this is certainly the star $\beta$ Per (Algol). And the beak of the bird (Cygnus) corresponds to the star $\beta$ Cyg (Albireo). These identifications are listed in Table 5. 


\begin{tabular}{|c|c|c|c|c|c|c|c|c|c|c|c|c|}
\hline \multirow[b]{2}{*}{ \# } & \multirow[b]{2}{*}{ Position } & \multirow[b]{2}{*}{ Star } & \multicolumn{4}{|c|}{ On the Farnese Atlas } & \multicolumn{4}{|c|}{ Star Position in 125 B.c. } & \multirow{2}{*}{\multicolumn{2}{|c|}{$\Delta \lambda\left(^{\circ}\right) \Delta \beta\left(^{\circ}\right.$}} \\
\hline & & & $\alpha\left(^{\circ}\right)$ & $\delta\left({ }^{\circ}\right)$ & $\lambda\left({ }^{\circ}\right)$ & $\beta\left(^{\circ}\right)$ & $\alpha\left(^{\circ}\right)$ & $\delta\left(^{\circ}\right)$ & $\lambda\left({ }^{\circ}\right)$ & $\beta\left(^{\circ}\right)$ & & \\
\hline 48 & Argo's s. rudder tip & $\alpha \mathrm{Car}$ & 77.4 & -49.4 & 62.7 & -71.9 & 84.4 & -52.7 & 75.7 & -76.1 & -13.0 & 4.2 \\
\hline 49 & CMa's mouth & $\alpha \mathrm{CMa}$ & 76.3 & -17.6 & 72.8 & -40.5 & 77.7 & -16.8 & 74.6 & -39.9 & -1.8 & -0.6 \\
\hline 50 & Corvus's beak & $\alpha \operatorname{Crv}$ & 152.3 & -16.6 & 161.0 & -26.2 & 155.7 & -13.2 & 162.8 & -21.7 & -1.8 & -4.5 \\
\hline 51 & Middle of Orion's belt & $\varepsilon$ Ori & 60.6 & -2.3 & 57.9 & -22.7 & 57.5 & -5.1 & 53.9 & -24.8 & 4.0 & 2.0 \\
\hline 52 & Hydra's eye & $\delta$ Hya & 108.8 & 5.5 & 109.6 & -16.9 & 100.7 & 10.7 & 100.8 & -12.6 & 8.8 & -4.3 \\
\hline 53 & Scorpius's sting & $\lambda$ Sco & 229.5 & -30.0 & 235.0 & -11.2 & 228.8 & -32.3 & 235.0 & -13.5 & -0.1 & 2.4 \\
\hline 54 & Middle of Sco's body & $\alpha$ Sco & 211.6 & -23.6 & 217.6 & -10.0 & 216.3 & -19.1 & 220.2 & -4.3 & -2.6 & -5.7 \\
\hline 55 & Tip of Sgr's arrow & $\gamma \mathrm{Sgr}$ & 234.5 & -29.0 & 239.1 & -9.1 & 238.0 & -27.3 & 241.7 & -6.7 & -2.6 & -2.4 \\
\hline 56 & Taurus's s. eye & $\alpha$ Tau & 46.7 & 12.2 & 47.6 & -5.3 & 39.6 & 9.6 & 40.2 & -5.7 & 7.4 & 0.4 \\
\hline 57 & Pisces, head of fish & $\gamma$ Psc & 315.9 & -6.6 & 316.4 & 10.0 & 322.0 & -7.4 & 321.9 & 7.3 & -5.5 & 2.7 \\
\hline 58 & N. tip Cap. rear horn & $\alpha$ Cap & 276.8 & -12.0 & 276.7 & 11.5 & 274.4 & -16.4 & 274.2 & 7.2 & 2.5 & 4.3 \\
\hline 59 & Medusa's head & $\beta$ Per & 29.3 & 28.6 & 37.4 & 15.4 & 15.5 & 30.9 & 26.7 & 22.2 & 10.7 & -6.8 \\
\hline 60 & Aries's muzzle & $\alpha$ Ari & -5.6 & 18.4 & 2.6 & 19.0 & 3.5 & 12.2 & 8.2 & 9.8 & -5.5 & 9.2 \\
\hline 61 & Andromeda's head & $\alpha$ And & 339.7 & 17.2 & 348.3 & 23.9 & 335.9 & 17.5 & 344.9 & 25.6 & 3.5 & -1.7 \\
\hline 62 & Pegasus's muzzle & $\varepsilon$ Peg & 302.5 & 10.2 & 307.5 & 29.7 & 299.8 & 1.8 & 302.4 & 22.2 & 5.1 & 7.5 \\
\hline 63 & Delphinus's head & $\alpha$ Del & 287.4 & 15.3 & 291.4 & 37.7 & 285.2 & 10.5 & 288.0 & 33.2 & 3.5 & 4.5 \\
\hline 64 & Ophiucus's head & $\alpha \mathrm{Oph}$ & 240.0 & 21.6 & 231.7 & 41.3 & 239.5 & 16.3 & 232.9 & 36.1 & -1.1 & 5.2 \\
\hline 65 & Hercules's head & $\alpha$ Her & 233.0 & 25.2 & 222.0 & 42.9 & 234.8 & 19.1 & 226.6 & 37.5 & -4.5 & 5.3 \\
\hline & Cassiopeia's breast & $\alpha$ Cas & 333.9 & 40.2 & 356.0 & 46.5 & 343.8 & 44.8 & 8.5 & 46.5 & -12.5 & 0.1 \\
\hline 67 & South edge of $\mathrm{CrB}$ & $\alpha \mathrm{CrB}$ & 224.5 & 33.5 & 207.8 & 47.7 & 211.3 & 35.4 & 192.6 & 44.5 & 15.2 & 3.2 \\
\hline 68 & Cygnus's beak & $\beta$ Cyg & 273.0 & 25.3 & 274.2 & 48.9 & 271.4 & 25.5 & 271.9 & 49.2 & 2.3 & -0.3 \\
\hline 69 & Cygnus's tail & $\alpha$ Cyg & 299.5 & 42.7 & 319.1 & 61.4 & 292.4 & 39.1 & 306.2 & 60.0 & 12.9 & 1.4 \\
\hline 70 & Centre of Lyra's shell & $\alpha \mathrm{Lyr}$ & 261.5 & 38.3 & 255.9 & 61.6 & 261.6 & 38.7 & 255.9 & 62.0 & 0.0 & -0.4 \\
\hline
\end{tabular}

On each of my pictures of the Farnese Atlas on which a given constellation figure is visible, I have measured its position and calculated its corresponding right ascension and declination. I have a total of 67 such positions for all 23 points. I have averaged together all the positions for each point, and the resulting right ascensions and declinations are presented in Table 5. I have also calculated the corresponding ecliptic coordinates, ${ }^{21}$ and these too are presented in Table 5.

The first column of Table 5 lists a running number, which is a continuation of the numbering from Table 3 . The items are ordered by increasing ecliptic latitude. The second column gives a verbal description of the constellation position selected, while the third column gives the modern name of the star at that position. The next two columns give the derived $\alpha$ and $\delta$ value in the reference frame of the Farnese Atlas, these being the observed positions. These equatorial positions are converted to ecliptic positions (longitude $\lambda$ and latitude $\beta$ ) in columns 6 and 7. Columns 8-11 give $\alpha, \delta, \lambda$ and $\beta$ of the modern stars as precessed back to the epoch of 125 B.C. Columns 12 and 13 are the differences between the observed and the model star positions in ecliptic coordinates.

The RMS scatter in the differences gives us a reasonable measure of the total uncertainties in the placements. The RMS scatter in the errors in ecliptic latitude is $4.1^{\circ}$. The deviations in ecliptic longitude will vary with ecliptic latitude by a factor of $1 / \cos (\beta)$ due to the convergence of the lines towards the pole. With this, I find that the RMS scatter of the errors in ecliptic longitude is $5 \% \cos (\beta)$. These errors have a good Gaussian distribution: $15 / 46=32 \%$ deviate by more than one-sigma, 
$1 / 46=2 \%$ deviate by more than two-sigma, while $12 / 23=52 \%$ and $11 / 23=48 \%$ deviate less than the average.

\section{A.2.4. Chi-square Analysis}

The best estimate for the date of the observations that were used to construct the constellations on the Farnese Atlas is that year for which the observed positions from Tables 3 and 5 most closely match the real sky. The optimal statistic for all such analyse ${ }^{22}$ is always the chi-square statistic, $\chi^{2}$.

The $\chi^{2}$ statistic is simply the summation over the data points of the squares of the deviations between the observations and the model in units of the standard deviation for the measurement. Symbolically, this is $\chi^{2}=\Sigma\left\{\left[\left(\mathrm{O}_{\mathrm{i}}-\mathrm{M}_{\mathrm{i}}\right) / \sigma_{\mathrm{i}}\right]^{2}\right\}$, where the summation is over all data items, the subscript ' $\mathrm{i}$ ' itemizes the datum, ' $\mathrm{O}$ ' is the observed value, ' $\mathrm{M}$ ' is the model value, and ' $\sigma$ ' is the measurement uncertainty. For example, for the first datum in Table 3, for an epoch of 125 B.C., the observed right ascension of the star $\gamma$ Ari is $0.5^{\circ}$, the model value is $0^{\circ}$ corresponding to the vernal equinox colure, and the typical scatter near the equator implies $\sigma=3.5^{\circ}$; so the $\chi^{2}$ contribution for this datum is 0.02 . For the first star on the equator (item \#13 in Table 1), the observed declination of $\sigma$ Ari in 125 B.C. is $4.7^{\circ}$, the model value is $0^{\circ}$ which corresponds to the equator, and the typical scatter is $\sigma=3.5^{\circ}$; so the $\chi^{2}$ contribution for this datum is 1.8. For item \#56 in Table 5, the observed ecliptic longitude of $\alpha$ Tau in 125 B.C. is $47.6^{\circ}$ on the Farnese Atlas, the model value is $40.2^{\circ}$, and the uncertainty near the equator is $5^{\circ}$; so the $\chi^{2}$ contribution for this datum is 2.2 . For a given date, the $\chi^{2}$ contributions can be summed over all data points to produce the $\chi^{2}$ value.

The $\chi^{2}$ value is smallest for the best model. In this case, the best model would be ideally that the Farnese Atlas represents the true positions of the identified stars for some particular date. By varying the date, the $\chi^{2}$ will vary also, with the date of the minimum $\chi^{2}$ being the best estimate date. The $68 \%$ probability error bar (i.e., the one-sigma uncertainty region) is that range of dates for which the $\chi^{2}$ is within 1.0 of the minimum value. With this, we have a standard technique for determining the best date for the Atlas as well as quantitatively deriving the real error bar in that date.

In Tables 3 and 5, I tabulate a total of 70 data points taken from the Farnese Atlas, and these constitute the observations. The model consists of the modern positions for these same stars or target circles as calculated by precession for various years. The $\chi^{2}$ contributions are simply the differences between the observed and model values (divided by the measurement uncertainty) squared. The uncertainties are $3.5^{\circ}$ in position for Table 3 and $5^{\circ}$ in ecliptic longitude for Table 5 (both with corrections for the latitude as needed). These are summed to give the $\chi^{2}$ value. I have calculated the $\chi^{2}$ for all dates from 400 B.C. to A.D. 200 at five-year intervals.

The date of minimum $\chi^{2}$ is 125 B.C. The $\chi^{2}$ at minimum is 66.3 .23 The $\chi^{2}$ rises to 67.3 for the years 70 B.C. and 180 B.C., so this is the one-sigma range for the date (i.e., there is a $68 \%$ probability that the real date is between 180 and 70 B.C.). The two-sigma range (i.e., the $95 \%$ confidence region) is that for which the $\chi^{2}$ is lower than $66.3+2^{2}=70.3$ and is between 245 and 10 B.C. The three-sigma range (i.e., the 
$99.7 \%$ confidence region) is that for which the $\chi^{2}$ is lower than $66.3+3^{2}=75.3$ and is between 305 B.C. and A.D. 50. The total size for the one-, two-, and three-sigma intervals is 110,235 , and 355 years respectively. It is encouraging and expected that these interval sizes are nearly in the proportions 1:2:3. The standard way to present such results is to quote the year of minimum $\chi^{2}$ as the best estimate of the date and to give the uncertainty as half the size of the one-sigma region. Thus, I conclude that the constellations on the Farnese Atlas were based on observations made in the year $125 \pm 55$ B.C.

It is appropriate here to note that this derived date is a very confident result. The techniques in the Appendices are all standard, straight forward and definitive. The deviations from the simple model are all Gaussian with zero outliers. The selection of various subsamples of the data still yields the same date (to within the quoted error bars), so the result is not simply due to some number of localized errors of any type. I cannot think of any astronomical effect or error nor of any historical vagary or error that would artificially produce the derived date. In all, to within the quoted error bars, the derived date of 125 B.C. is of high confidence.

\section{ACKNOWLEDGEMENTS}

I thank Maurizio Paolillo and Guy Consolmagno for help with access to the Farnese Atlas for photography; the National Museum in Naples for granting me access to the statue for taking photographs; Roger Macfarlane and Paul Mills for access to their translation of Hipparchus's Commentary before publication; and Eric Seidel for help in translations.

\section{REFERENCES}

1. The best pictures of the Atlas appear in H. Stern, "Classical Antiquity" in the entry titled "Astronomy and astrology" in Encyclopedia of world art (New York, 1960), ii, cols 48-57 and plate 23. Many short descriptions of the statue have appeared, of which the best are G. Aujac, "The foundations of theoretical cartography in archaic and classical Greece", in History of cartography, ed. by J. B. Harley and D. Woodward (Chicago, 1987), 142-3; J. Evans, The history \& practice of ancient astronomy (New York, 1998), 78-79; M. Fiorini, Sfere terrestri e celesti (Rome, 1899), 8-25; C. Gialanella and V. Valerio, "Atlas Farnese" in Cartes et figures de la Terre (Paris, 1980), 84; E. L. Stevenson, Terrestrial and celestial globes (New Haven, 1921), 1-25; G. Thiele, Antike Himmelsbilder (Berlin, 1898), 27-45; and D. J. Warner, The sky explored, celestial cartography 1500-1800 (New York, 1979), 278-9. A detailed history of the historical writings on the Farnese Atlas appears in V. Valerio, "Historiographic and numerical notes on the Atlante Farnese and its celestial sphere", Der Globusfreund, xxxv/xxxvii (1987), 97-124.

2. Here is the complete list of constellations visible on the Farnese Atlas in the constellation order as given in the Almagest: Dra, Cep, Boo, CrB, Her, Lyr, Cyg, Cas, Per, Aur, Oph, Ser, Aql, Del, Peg, And, Ari, Tau, Gem, Cnc, Leo, Vir, Lib, Sco, Sgr, Cap, Aqr, Psc, Cet, Ori, Eri, Lep, CMa, Argo, Hya, Crt, Crv, Cen, Lup, Ara, and CrA. (Stevenson cites 42 constellations while Gialanella and Valerio [and hence Aujac] cite 43 constellations.) There are a number of constellations from Antiquity that are missing. Ursa Major is missing as it is located entirely within the hole in the top of the globe. Canis Minor is entirely positioned under one of the hands holding the globe. Piscis Austrinus is completely hidden by the shoulder of the Titan. Of the older Greek constellations, the only ones missing without cause are Ursa Minor, Triangulum, and Sagitta. 
A short, simple line segment near a wing of Cygnus could conceivably be Sagitta, but this has the strong iconography, the wrong orientation, and is $15^{\circ}$ misplaced, and so I conclude that the line segment is not Sagitta. The later Greek constellations of Equuleus, Coma Berenices, and Antinous are all missing and would certainly have been visible if depicted. The only addition that is not part of the set of old constellations is a curious small rectangular structure with three internal lines that appears above the Crab. This is certainly not any known constellation, and the only explanation suggested is that is this represents the comet known as "Throne of Caesar" or a throne of Zeus (Stern, op. cit. (ref. 1); Thiele, op. cit. (ref. 1)).

3. Stern, op. cit. (ref. 1), col. 49.

4. B. E. Schaefer, "Latitude and epoch for the origin of the astronomical lore of Eudoxus", Journal for the history of astronomy, xxxv (2004), 161-223.

5. Aratus Phaenomena, transl. by D. Kidd (Cambridge, 1997). Another widely available translation is by G. R. Mair and appears in the Loeb Classical Library series titled Aratus (Cambridge, MA, 1921) with many subsequent reprints.

6. R. T. Macfarlane and P. S. Mills, Hipparchus' commentaries on the Phaenomena of Aratus and Eudoxus, manuscript, 2003. This translation is the first in English and the first in modern times.

7. T. Condos, Star myths of the Greeks and Romans: A sourcebook (Grand Rapids, MI, 1997).

8. G. J. Toomer, Ptolemy's Almagest (Princeton, 1998).

9. That is, one of the primary statements by Aratus on the cardinal points of the ecliptic is true for a time almost a millennium earlier. This immediately tells us that Aratus (and hence Eudoxus) include lore that was already ancient for their times. A detailed analysis of the lore in Aratus and Eudoxus (in Schaefer, op. cit. (ref. 4)) shows that all the lore is from many centuries before even Homer and Hesiod. A detailed analysis gives a date of $1130 \pm 80$ B.C.

10. B. E. Schaefer, "Latitude and epoch for the formation of the southern Greek constellations", Journal for the history of astronomy, xxxiii (2002), 313-50. Roughly, the extinction angle plus refraction correction for the bright stars in Centaurus, Crux, and Carina is close to $2^{\circ}$, while the gap for these stars (the angle above the region of invisibility) is certainly greater than $1^{\circ}$ and more likely around $2^{\circ}$ (although it could be larger). Thus, the correction to the simply derived latitude is likely $4^{\circ}$, but the value could be $3^{\circ}$ or larger.

11. Evans, op. cit. (ref. 1), 91-95.

12. Schaefer, op. cit. (ref. 4), Table 7 and Section 6.7 .

13. Stevenson, op. cit. (ref. 1), 1-25; Evans, op. cit. (ref. 1), 78-79 and Figure 5.11.

14. The quote is from O. Neugebauer, A history of ancient mathematical astronomy (3 vols, Berlin, 1975), 277-80; Evans, op. cit. (ref. 1), 103.

15. K. A. Pickering, "Evidence of an ecliptical coordinate basis in the Commmentary of Hipparchos", Dio, ix (1999), 26-29.

16. D. W. Duke, "Hipparchus' coordinate system", Archive for the history of exact sciences, lvi (2002), 427-33; J. B. J. Delambre, Histoire de l'astronomie ancienne (1817; repr. New York, 1965), i, $117,172,184$.

17. A history of this dispute is given in B. E. Schaefer, "The great Ptolemy-Hipparchus debate", Sky \& telescope, ciii (2002), Feb. issue, 38-44.

18. H. Vogt, "Versuch einer Wiederstellung von Hipparchs Fixsternverzeichnis", Astronomische Nachtrichten, ccxxiv (1925), cols 2-54; G. Grasshoff, The history of Ptolemy's star catalogue (New York, 1990); N. M. Swerdlow, “The enigma of Ptolemy's catalogue of stars”, Journal for the history of astronomy, xxiii (1992), 173-83; and D. W. Duke, "The depth of association between the ancient star catalogues", Journal for the history of astronomy, xxxiv (2003), 227-30.

19. The obliquity of the ecliptic for 125 B.C. is $23.71^{\circ}$, as determined from J. Meeus, Astronomical algorithms (Richmond, VA, 1991), 135-6. The mean obliquity of the ecliptic is $23.76^{\circ}$ in 500 B.C. and $23.70^{\circ}$ in 1 A.D.

20. Meeus, op. cit. (ref. 19), 126-8. Proper motions of all stars used in this paper are completely negligible.

21. The conversion from ecliptic to equatorial coordinates is taken from Equations 12.3 and 12.4 in 
Meeus, op. cit. (ref. 19), 87-92.

22. Technically, the chi-square analysis applies only when the errors have Gaussian distributions. However, in practice, the chi-square technique is still best even for distributions far from Gaussian, even though the derived error bars become more uncertain. Fortunately, the residuals for the analysis in this paper are all good Gaussian distributions.

23. With 70 data items and one variable fit parameter, the total number of degrees of freedom in this chi-square fit is 69 . Then, the reduced chi-square is $66.3 / 69=0.96$. In general, a reduced chisquare of near unity implies a good fit. However, this conclusion of a good fit does not apply for this case because the size of the uncertainties were selected as the observed scatter which then forces a reduced chi-square of near unity. If alternatively, we had chosen a substantially larger or smaller value for the sigma, then the derived reduced chi-square would have been greatly smaller or larger than unity respectively. If we had any way of independently knowing the typical total uncertainty (which we do not), then we could have used this value and derived a meaningful reduced chi-square that is useful for evaluating the quality of the model. One comforting fact is that the typical scatters (which yield a reduced chi-square near unity) have reasonable sizes, for example judged based on the typical scatters for the lore of Eudoxus (see Schaefer, op. cit. (ref. 4)). The determination of the best date does not depend on any scaling of the adopted uncertainties. However, the derived error bars for the best-fit epoch will depend on the size of the adopted uncertainties for the individual data points. And, indeed, the process can be turned around so as to derive the typical total uncertainty of a single point (based on the average uncertainty so as to make the reduced chi-square near unity). With this derived uncertainty for an individual observation, we will necessarily get an acceptable reduced chi-square and the derived error bars on the epoch will be good. In all, the exact value of the reduced chi-square is not meaningful since the uncertainties have been taken from the observed scatter in the data themselves, but this choice allows for valid error bars on the date. 